\title{
Autoeficacia docente para la convivencia escolar en la formación inicial docente
}

\author{
Darío F. Rojas-Díaz y Oscar R. Nail \\ Facultad de Educación, Universidad de Concepción, Víctor Lamas 1290, Concepción, Chile \\ (correo-e: dariorojas@udec.cl, onail@udec.cl)
}

Recibido Jun. 10, 2021; Aceptado Ago. 2, 2021; Versión final Oct. 11, 2021, Publicado Feb. 2022

\begin{abstract}
Resumen
Esta investigación tiene como objetivo diseñar una escala de Autoeficacia Docente para la Convivencia Escolar (ADCE) en la formación inicial docente. La autoeficacia es un constructo psicológico que permite evaluar de forma prospectiva la capacidad de los profesores para enfrentar los desafíos de la convivencia escolar que requieren una alta motivación, perseverancia e iniciativa. El instrumento fue aplicado a una muestra de 115 estudiantes de pedagogía de pregrado de la Universidad de Concepción, Chile. El diseño incluyó la validación cualitativa de estudiantes y expertos, mientras que la validación cuantitativa se realizó mediante un análisis factorial exploratorio. Los resultados permitieron evidenciar tres factores latentes (inclusión, colaboración y democracia) que explican el $56.5 \%$ de la varianza con índices de ajuste y coeficientes de fiabilidad adecuados. En conclusión, el instrumento y los factores encontrados son parsimoniosos con el constructo teórico utilizado y los resultados concuerdan con otros estudios similares.
\end{abstract}

Palabras clave: autoeficacia; convivencia escolar; formación inicial docente; democracia; inclusión

\section{Schoolwide climate self-efficacy teaching for pre-service teachers}

\begin{abstract}
This study aims to design a self-efficacy teaching scale for schoolwide climate (ADCE, in Spanish) for preservice teachers. Self-efficacy is a psychological construct that allows making prospective assessments of teacher's abilities in school environments that require high motivation, perseverance, and initiative. The instrument was applied to a sample of 115 undergraduate pedagogy students enrolled at the University of Concepción, Chile. The design includes qualitative validations of students and experts and a quantitative validation assessment performed by exploratory factor analysis. The results show three latent factors (inclusion, collaboration, and democracy) that explain 56.5\% of the variance with fit indices and with adequate reliability coefficients. In conclusion, the instrument and the factors examined are parsimonious with the theoretical construct applied and the results are in concordance with findings of similar studies.
\end{abstract}

Keywords: self-efficacy; schoolwide climate; preservice teachers; democracy; inclusion 


\section{INTRODUCCIÓN}

La preocupación mundial por los temas relacionados a la convivencia en la educación fue impulsada, principalmente, por el Informe de Delors (1996), documento que propone los cuatro pilares de la educación: aprender a conocer, aprender a hacer, aprender a ser y aprender a vivir juntos. Dentro de esa propuesta, aprender a vivir juntos es considerado parte de los aprendizajes esenciales para lograr una educación que fomente el conocimiento de los otros, su cultura y espiritualidad durante toda la vida. En ese marco, se puede considerar que la convivencia escolar se relaciona mayormente con el pilar "Aprender a vivir juntos". El estudio de la convivencia escolar ha sido abordado desde distintas perspectivas metodológicas y teóricas. Estos estudios recibieron el aporte desde diversos enfoques, razón por la cual la convivencia escolar, como objeto de estudio, se fue complejizando paulatinamente a lo largo del tiempo sin una dirección concreta. Además, la convivencia escolar es una denominación que solo es usada en Latinoamérica y España, por lo que no tiene una traducción directa fuera de estas regiones (Muñoz et al., 2018), lo que hace más complejo su estudio y comparativa.

Así, a pesar de los esfuerzos de la comunidad científica por realizar una aclaración teórica respecto al concepto de convivencia escolar en Iberoamérica (Ascorra et al., 2018; Muñoz et al., 2018; Retuert y Castro, 2017), aún persiste la dispersión y ensamblaje sincrético de los conceptos que impiden una profundización de los estudios (Fierro-Evans y Carbajal-Padilla, 2019), a la vez que su naturaleza subjetiva dificulta su evaluación. Una situación similar es la que se puede observar fuera de Iberoamérica, donde la convivencia escolar recibe la denominación homóloga de clima escolar con perspectiva global o School-wide Climate (Muñoz et al., 2018). Esta denominación anglosajona aún se encuentra en proceso de consensuar su conceptualización y, también comparte las mismas dificultades para su evaluación (Charlton et al., 2021). Por ello, no es posible abordar la convivencia escolar como un fenómeno único, acabado y con límites definidos, sino que es necesario un enfoque de aproximaciones sucesivas (Chaparro et al., 2015) que permita abarcar aspectos cada vez más específicos, pero que, a la vez, vayan ampliando y profundizando su entendimiento.

En la misma línea, Fierro-Evans y Carbajal-Padilla (2019) presentan una revisión actualizada del concepto de Convivencia Escolar, definiéndola como "los procesos y resultados del esfuerzo por construir una paz duradera entre los miembros de la comunidad escolar, a partir de prácticas pedagógicas y de gestión: inclusivas, equitativas y participativas que aborden de manera constructiva el conflicto" (p. 13). En este sentido, el rol del profesor es ser un mediador entre los estudiantes y el proceso de convivencia, pues es él quien, en última instancia, facilita el desarrollo de conocimientos, habilidades y actitudes de los estudiantes al realizar una traducción de los objetivos curriculares, sociales y culturales del sistema educativo en función de sus propias competencias pedagógicas y de gestión (Retuert y Castro, 2017). El proceso que permite desarrollar y certificar tales competencias en instituciones especializadas se denomina Formación Inicial Docente (FID), un proceso que busca vincular estratégicamente la preparación profesional con los desafíos educativos contextuales y contemporáneos de su entorno (Loubiès, Valdivieso y Vásquez, 2020), mediante programas formadores que catalizan la educación en la sociedad y les permitan comprender el clima cultural y social (Pedraja-Rejas et al., 2021). En consecuencia, como todo proceso de formación, se hace necesario evaluar prospectivamente los diversos aspectos de la FID, en específico aquellos aspectos relacionados con las tareas que requieren mayor esfuerzo, persistencia y motivación, tales como la educación democrática, la gestión de la diversidad y la colaboración, pues revisiones de estudios recientes han indicado que existe una necesidad de aumentar la sistematización e instrumentalización psicométrica de estos aspectos (Charlton et al., 2021).

En este contexto, la autoeficacia es un constructo psicológico derivado de la teoría del aprendizaje sociocognitivo de Bandura (1977) que puede ser usada para la evaluación prospectiva de las capacidades de las personas. La autoeficacia se basa en la autoevaluación de las creencias acerca de las capacidades propias de una persona y no depende directamente de los conocimientos adquiridos, más bien asume que los individuos evalúan sus propias experiencias de tal forma que las creencias acerca de sus capacidades y logros previos ejercen influencia en las actuaciones futuras (Dunn y Rakes, 2011). Por este motivo, la autoeficacia puede considerarse predictor del comportamiento futuro, puesto que la evidencia experimental muestra que una mayor autoeficacia sobre una capacidad específica deriva en un mejor desempeño de esta (Pajares y Schunk, 2002; Lazarides et al., 2021).

En específico, desde la década de los noventa se ha estudiado un tipo de autoeficacia particular denominada autoeficacia docente (Tschannen-Moran y Hoy, 2001). Este constructo evalúa la creencia que el profesor tiene de sus capacidades para influir de forma positiva en el proceso de aprendizaje de los estudiantes, incluso en condiciones desfavorables para llevar a cabo su labor (Covarrubias y Mendoza, 2016). Sin embargo, estos trabajos no están directamente dirigidos a evaluar la autoeficacia para la convivencia escolar y tampoco están enmarcados dentro de la formación inicial docente, ya que, aunque la autoeficacia docente aborda elementos disciplinarios y de manejo de la sala de clases, no incluye dimensiones específicas relativas a la democracia 
y la inclusión, los que se consideran relevantes para el concepto de convivencia escolar utilizado en Iberoamérica (Chaparro et al., 2015; Fierro-Evans y Carbajal-Padilla, 2019).

Para definir un marco conceptual que permita delimitar el sentido de autoeficacia para la convivencia escolar que se desea evaluar en los docentes en formación, se establecieron dos dimensiones o dominios principales: la dimensión pedagógica y la dimensión democrática. La dimensión pedagógica se relaciona con el direccionamiento de la actividad del aula para guiar el desarrollo de las capacidades particulares de cada estudiante mediante la persecución y valoración de fines comunes, considerando a la convivencia como el proceso que ocurre en un espacio formativo de la vida escolar (Fierro-Evans y Carbajal-Padilla, 2019). Mediante esta dimensión se busca evaluar la autopercepción de la capacidad del futuro docente para promover el reconocimiento y valoración de la diversidad mediante el desarrollo de actividades, principalmente colaborativas, haciendo énfasis en la conformación de grupos heterogéneos en lo social y cultural. Por otro lado, la dimensión Democrática consiste en la capacidad para mantener una actitud positiva a favor de los estudiantes y el proceso educativo que lleva a cabo, es decir, en el sentido de Dewey (1998), la acción del docente debe referir a la acción de los estudiantes y viceversa como una forma de dar dirección a la educación en la búsqueda de un resultado común, manteniendo el respeto y la flexibilidad para atender las iniciativas de los estudiantes utilizando, idealmente, procesos deliberativos.

La autoeficacia se presenta como un constructo psicológico adecuado para evaluar las dimensiones consideradas, pues posee tres características que guardan relación con la naturaleza de lo que se desea abordar. De acuerdo con las definiciones de Covarrubias y Mendoza (2013), las tres características de la autoeficacia consideradas son: 1) dinámica, 2) cíclica y 3) específica de un dominio. La primera característica considera a la autoeficacia como una percepción que cambia debido a las nuevas experiencias vividas por las personas, por lo que su evaluación posibilita el análisis longitudinal del efecto de potenciales intervenciones para mejorar y monitorear la autoeficacia en los dominios considerados. La segunda característica contempla que los individuos son producto y productores de sus circunstancias, por lo que, además de considerar la agencia del profesor en los procesos de convivencia, también se debe considerar que la convivencia y los climas producidos por esta pueden tener efecto en la autoeficacia de los profesores (Guangbao y Timothy, 2021). Por último, la tercera característica establece que no existe la autoeficacia en todos los contextos de desempeño humano, sino que debe ser definida en un dominio específico, lo que es adecuado para los objetivos de esta investigación, pues se busca evaluar el dominio específico de la formación inicial docente para la convivencia escolar en las dimensiones descritas con anterioridad.

Debido a que la convivencia escolar es una construcción en la que es partícipe toda la comunidad educativa, es necesario que los futuros profesores sean propensos a mantener sus niveles de motivación y perseverancia cuando las condiciones sean desfavorables. Por este motivo, la autoeficacia es un constructo adecuado para la evaluación prospectiva de las capacidades de los futuros docentes, ya que tiene efecto en el comportamiento, sobre todo en los procesos motivacionales y de selección que influyen en la perseverancia en condiciones desfavorables (Milson y Mehlig, 2002). En los procesos motivacionales, la autoeficacia influye en la autorregulación emocional cognitiva, por lo que, afecta a las atribuciones que hacen los sujetos de los factores externos o internos al éxito. Por ello, un profesor en formación con una percepción de alta autoeficacia propenderá a creer que el éxito depende más de él mismo que de los factores externos o las dificultades de su labor, por lo que tendrá una tendencia a mantener una mayor motivación.

En el caso de los procesos de selección, la autoeficacia tiene efecto en las elecciones que hacen las personas en su vida, pues estas tienden naturalmente a evitar actividades o tomar decisiones que crean que pueden llevarlos al fracaso. Así, un profesor en formación con alta autoeficacia tendrá una iniciativa más alta de salir de su zona de confort y mantener un mayor compromiso con las metas que se fija. Tomando en consideración todo lo anterior, la presente investigación tuvo por objetivo el diseño de un instrumento que permita evaluar la autoeficacia docente de los futuros profesores relacionada a la convivencia escolar en la formación inicial docente.

\section{METODOLOGÍA}

La investigación se corresponde con el diseño exploratorio, no-experimental, descriptivo, correlacional y transversal con métodos prominentemente cuantitativos.

\section{Muestra}

Los participantes corresponden a una muestra no-probabilística e intencionada, pues la población objetivo consistió en estudiantes de pedagogía de pregrado de la Universidad de Concepción, Chile. La edad media de los participantes fue de $M=23.2$ años ( $D T=2.8$ ), con una formación universitaria promedio de $M=3.8$ ( $D T=$ 1.2) años. La frecuencia de la distribución de géneros autoreportada fue de 74 mujeres, 40 hombres y 3 
participantes con otro tipo de género. La participación por carrera profesional o disciplina fue de 25 para Educación Diferencial, 35 para Pedagogía en Inglés, 34 para Pedagogía en Matemáticas y 21 de Otras disciplinas.

\section{Instrumentos}

Se diseñó una escala de Autoeficacia Docente para la Convivencia Escolar (ADCE) con ítems conformados por preguntas de autoeficacia percibida de la capacidad para promover, planificar y evaluar actividades principalmente colaborativas, haciendo énfasis en la conformación de grupos heterogéneos. Para la confección de las preguntas se utilizaron como referencia las escalas: Character Education Efficacy Belief Instrument (Milson y Mehlig, 2002), Teacher efficacy (Gibson y Dembo, 1964), School-wide Climate Scale (Muñoz et al., 2018) y Convivencia Escolar Democrática (Chaparro et al., 2015). La escala diseñada fue sometida a la evaluación del contenido por estudiantes de pedagogía y una revisión de dos expertos, más un análisis factorial exploratorio para determinar las variables latentes. El instrumento fue aplicado en forma online mediante una plataforma específicamente diseñada para esta investigación y los análisis estadísticos se realizaron a través del software $\mathrm{R}$ (https://www.r-project.org).

\section{RESULTADOS}

Se comenzó el proceso de diseño con una escala inicial de 63 ítems conformados por preguntas sobre la capacidad autopercibida para organizar y promover una convivencia escolar democrática. El instrumento inicial se sometió a una lectura y grupo de discusión conformado por 4 estudiantes de pregrado de pedagogía quienes leyeron los ítems y dieron su opinión sobre la claridad de las preguntas y los temas que trataban. En este proceso, algunas preguntas fueron consideradas redundantes y otras trataban temas que no eran de su conocimiento, por lo que se redactaron nuevamente y fueron evaluadas por dos expertos en el área de convivencia escolar, quienes revisaron la redacción y pertinencia de las preguntas al constructo. Esta evaluación dio lugar a un instrumento de 22 preguntas, las que componen el instrumento a evaluar en la muestra intencionada, con 13 preguntas de la dimensión pedagógica (ítems de 2 a 9 y de 18 a 21, alfa de Cronbach $=0.9$ ) y 9 preguntas de la dimensión democrática (ítems 1 y de 10 a 17 , alfa de Cronbach $=0.844$ ). Cada pregunta se evaluó en una escala con valores entre 1 y 6 , con $1=$ "no soy capaz" y $6=$ "soy totalmente capaz".

\section{Análisis Factorial Exploratorio}

Para evaluar el instrumento, se realizó un análisis factorial exploratorio de las respuestas de los estudiantes. Todos los ítems evaluados presentaron normalidad univariada según la prueba de Kolmogorov-Smirnov. Sin embargo, la prueba de Mardia arrojó que la distribución multivariada no era normal ( $p<0.001)$, por lo que, para los análisis posteriores, se utilizó la matriz de correlaciones policóricas.

Con respecto a la factibilidad de la aplicación del análisis factorial, se obtuvo que el determinante de la matriz de correlaciones fue cercano a cero $\left(4.5 \times 10^{-8}\right)$ y la medida de adecuación muestral de Kaiser-Meyer-Olkin fue $\mathrm{KMO}=0.827$. Además, la prueba de esfericidad de Bartlett mostró que existen diferencias significativas entre la matriz identidad y la matriz de correlaciones $\left(X^{2}=1790.4, p<0.001\right)$, por lo que se puede argumentar que existe suficiente correlación entre los ítems para realizar un análisis factorial.

Para determinar la cantidad de factores latentes, se realizó un análisis paralelo de Horn, se utilizó el índice Very Simple Structure (Revelle y Rocklin, 1979) y el algoritmo Wayne Velicer's MAP (Velicer, 1976). Todos estos métodos sugirieron mantener la cantidad de 3 factores latentes. En el proceso de extracción, se utilizaron 3 factores fijos y se tomaron en cuenta 21 de los 22 ítems que alcanzaron cargas factoriales superiores a $|0.4|$. Así, mediante el método de extracción por ejes principales, la solución factorial obtuvo los índices RMSR=0.07 y RMSEA=0.131.

Debido a que en la teoría se considera que los factores están relacionados entre ellos, se realizó una rotación oblicua oblimin y se extrajeron las cargas factoriales mostradas en la Tabla 1, donde los ítems 1 a 9 conforman el primer factor denominado Inclusión, los ítems 10 a 17 conforman el segundo factor denominado Democracia y los ítems 18 a 21 el tercer factor denominado Colaboración. La solución factorial y sus tres factores explican el $56.5 \%$ de la varianza, la que se distribuye proporcionalmente por cada factor y en forma acumulada según se muestra en la Tabla 2. La correlación entre estos factores se muestra en la Tabla 3, donde se puede apreciar que la mayor correlación es alcanzada por los factores Inclusión y Democracia, mientras que la menor correlación se encuentra entre los factores Inclusión y Colaboración. Con respecto a la fiabilidad del instrumento con la muestra, el coeficiente alfa de Cronbach para todos los ítems fue de 0.921 , mientras que el alfa de Cronbach de los ítems que componen cada factor fue de $0.893,0.833$ y 0.821 para los fatores Inclusión, Democracia y Colaboración respectivamente. 
Con respecto a las variables que caracterizan la muestra, no se encontraron correlaciones significativas entre la edad y los años de formación universitaria. Sin embargo, se pudo evidenciar una correlación significativa entre la edad y la suma de puntajes del factor Inclusión $(r=-0.254, p<0.01)$, Democracia $(r=-0.189, p<0.05)$ y Colaboración $(r=-0.249, p<0.01)$. Estas correlaciones aumentan si se controla la variable de años de formación universitaria y se calculan las correlaciones parciales de edad con respecto a Inclusión ( $r=-0.328)$, Democracia $(r=-0.215)$ y Colaboración $(r-0.292)$.

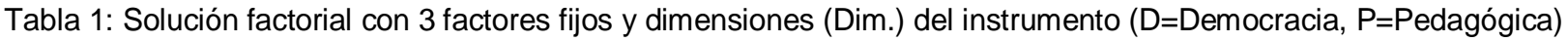

\begin{tabular}{|c|c|c|c|c|c|}
\hline$\#$ & Dim. & Ítem & $f 1$ & $f 2$ & f3 \\
\hline 1 & $\mathrm{D}$ & $\begin{array}{l}\text { Utilizar sistemáticamente procesos deliberativos como parte del proceso educativo de } \\
\text { los estudiantes }\end{array}$ & $\mid 0.407$ & & \\
\hline 2 & $P$ & $\begin{array}{l}\text { Reconocer diferencias y similitudes entre las distintas realidades socioculturales } \\
\text { existentes en la comunidad educativa }\end{array}$ & $\mid 0.475$ & & \\
\hline 3 & $P$ & $\begin{array}{l}\text { Organizar grupos de aprendizaje con estudiantes que provengan de diferentes } \\
\text { realidades socioculturales }\end{array}$ & $\mid 0.551$ & & \\
\hline 4 & $P$ & $\begin{array}{l}\text { Promover la reflexión de los estudiantes sobre las razones por las cuales ellos incluyen } \\
\text { o excluyen a compañeros de los grupos }\end{array}$ & 0.595 & & \\
\hline 5 & $P$ & $\begin{array}{l}\text { Implementar actividades de aprendizaje donde las ideas de todos los estudiantes sean } \\
\text { importantes para alcanzar los objetivos }\end{array}$ & $\mid 0.615$ & & \\
\hline 6 & $P$ & $\begin{array}{l}\text { Promover un ambiente socioeducativo en el aula lo suficientemente cercano a la realidad } \\
\text { sociocultural de los estudiantes }\end{array}$ & $\mid 0.650$ & & \\
\hline 7 & $P$ & $\begin{array}{l}\text { Desarrollar actividades para que los estudiantes valoren la diversidad de opiniones como } \\
\text { una oportunidad para mejorar sus propios aprendizajes }\end{array}$ & $\mid 0.776$ & & \\
\hline 8 & $P$ & $\begin{array}{l}\text { Reconocer y mejorar experiencias de aprendizaje donde los estudiantes se sientan } \\
\text { excluidos, aislados o rechazados por compañeros/as }\end{array}$ & $\mid 0.799$ & & \\
\hline 9 & $P$ & $\begin{array}{l}\text { Propiciar oportunidades en la clase para la reflexión y análisis del contexto sociocultural } \\
\text { donde se realiza la actividad educativa }\end{array}$ & || $\mid 0.873$ & & \\
\hline 10 & D & $\begin{array}{l}\text { Atender y apoyar a los estudiantes, con independencia de su historial de conducta o } \\
\text { académico }\end{array}$ & & 0.465 & \\
\hline 11 & $\mathrm{D}$ & $\begin{array}{l}\text { Cumplir las normas de convivencia acordadas democráticamente, aunque estas no sean } \\
\text { de mi agrado. }\end{array}$ & & 0.475 & \\
\hline 12 & $\mathrm{D}$ & $\begin{array}{l}\text { Ser flexible en los temas de la asignatura, atendiendo la curiosidad de los estudiantes, } \\
\text { aunque deba alejarme de lo planificado }\end{array}$ & & 0.527 & \\
\hline 13 & $\mathrm{D}$ & Mantener el respeto por los estudiantes, aunque ellos me falten el respeto a mí & & 0.572 & \\
\hline 14 & $\mathrm{D}$ & Escuchar y atender las iniciativas de los estudiantes, aunque estas no sean de mi agrado. & & 0.719 & \\
\hline 15 & $\mathrm{D}$ & $\begin{array}{l}\text { Modificar mi propia conducta tomando en cuenta la opinión de los estudiantes, si la } \\
\text { situación lo amerita }\end{array}$ & & 0.727 & \\
\hline 16 & $\mathrm{D}$ & $\begin{array}{l}\text { Mantener, a pesar de posibles dificultades en la convivencia, expectativas positivas hacia } \\
\text { los estudiantes }\end{array}$ & & 0.847 & \\
\hline 17 & $\mathrm{D}$ & Mantener una conducta de respeto ante situaciones de convivencia adversas & & 0.864 & \\
\hline 18 & $\mathrm{D}$ & Evaluar el trabajo grupal, considerando la contribución individual de los miembros & & & 0.436 \\
\hline 19 & $\mathrm{P}$ & $\begin{array}{l}\text { Conformar grupos colaborativos de estudiantes donde los miembros tienen destrezas y } \\
\text { habilidades diferentes }\end{array}$ & & & 0.654 \\
\hline 20 & $\mathrm{P}$ & Ayudar a conformar grupos colaborativos de estudiantes socialmente heterogéneos & & & 0.707 \\
\hline 21 & $\mathrm{P}$ & Conformar grupos colaborativos con miembros que se desconocen entre ellos & & & 0.852 \\
\hline
\end{tabular}

Tabla 2: Varianza explicada por cada factor

\begin{tabular}{|l|c|c|c|}
\hline & Inclusión & Democracia & Colaboración \\
\hline Varianza Proporcional & $23.3 \%$ & $20.7 \%$ & $12.5 \%$ \\
\hline Varianza Proporcional Acumulada & $23.3 \%$ & $44.0 \%$ & $56.5 \%$ \\
\hline
\end{tabular}

Tabla 3: Correlación entre factores

\begin{tabular}{|l|c|c|c|}
\hline & Inclusión & Democracia & Colaboración \\
\hline Inclusión & 1.00 & 0.55 & 0.51 \\
\hline Democracia & 0.55 & 1.00 & 0.45 \\
\hline Colaboración & 0.51 & 0.45 & 1.00 \\
\hline
\end{tabular}

En lo referente a las diferencias de puntajes de los factores según género, estos se muestran en la Tabla 3 , se encontraron diferencias significativas entre los géneros femenino y masculino para los factores Inclusión con $\mathrm{t}(110)=2.824, \mathrm{p}<0.05$, Democracia con $\mathrm{t}(110)=2.854, \mathrm{p}<0.05$, y Colaboración con $\mathrm{t}(110)=2.187, \mathrm{p}<0.05$. 
Para esta variable, el género femenino tuvo diferencias positivas de la media del puntaje sobre el género masculino de 2.491, 2.428 y 1.490 para los factores Inclusión, Democracia y Colaboración respectivamente.

Para el caso de las disciplinas encuestadas, los puntajes para cada factor se muestran en la Tabla 4. El análisis de la varianza de un factor reveló que existen diferencias significativas entre las medias de la puntuación del factor Inclusión $F(3,111)=3.630, p<0.05$. Las comparaciones múltiples mostraron que existen diferencias estadísticamente significativas para el factor Inclusión entre las disciplinas de Educación Diferencial y Pedagogía en Inglés $\mathrm{t}(111)=2.704, \mathrm{p}<0.05$; y entre Educación Diferencial y Pedagogía en Matemáticas $\mathrm{t}(111)=3.025, \mathrm{p}<0.05$. En ambos casos, la diferencia de la media del puntaje fue a favor de Educación Diferencial. Por otro lado, no se encontraron diferencias significativas entre los otros pares de disciplinas comparadas para ninguno de los otros factores.

Tabla 3: Edad, Años de Formación y Puntaje por Género

\begin{tabular}{|l|r|r|r|r|r|r|}
\hline & & Edad & Años de Formación & Inclusión & Democracia & Colaboración \\
\hline Femenino & $\mathrm{M}$ & 22,7 & 3,9 & 45,5 & 43,5 & 19,6 \\
\hline & $\mathrm{DT}$ & 2,6 & 1,1 & 5,9 & 3,9 & 3,2 \\
\hline Masculino & $\mathrm{M}$ & 24,3 & 3,8 & 42,0 & 41,1 & 18,2 \\
\hline & $\mathrm{DT}$ & 3,1 & 1,4 & 6,8 & 4,9 & 3,9 \\
\hline Otro & $\mathrm{M}$ & 24,3 & 3,8 & 42,0 & 41,1 & 18,2 \\
\hline & $\mathrm{DT}$ & 3,1 & 1,4 & 6,8 & 4,9 & 3,9 \\
\hline
\end{tabular}

Tabla 4: Edad, Años de Formación y Puntaje por Disciplina

\begin{tabular}{|l|r|r|r|r|r|r|}
\hline & & Edad & Años de Formación & Inclusión & Democracia & Colaboración \\
\hline Educación Diferencial & $\mathrm{M}$ & 22,4 & 4,1 & 47,4 & 44,6 & 20,5 \\
\hline & $\mathrm{DT}$ & 1,4 & 0,3 & 5,5 & 4,5 & 3,1 \\
\hline Pedagogía en Inglés & $\mathrm{M}$ & 24,2 & 4,4 & 42,9 & 41,6 & 18,1 \\
\hline & $\mathrm{DT}$ & 3,2 & 1,5 & 6,4 & 4,5 & 3,9 \\
\hline Pedagogía en Matemática & $\mathrm{M}$ & 23,8 & 3,9 & 42,3 & 41,7 & 18,3 \\
\hline & $\mathrm{DT}$ & 3,2 & 0,9 & 6,5 & 4,8 & 3,4 \\
\hline Otras disciplinas & $\mathrm{M}$ & 21,8 & 2,5 & 44,8 & 42,5 & 20,1 \\
\hline & $\mathrm{DT}$ & 2,2 & 1,1 & 6,9 & 3,8 & 2,5 \\
\hline
\end{tabular}

\section{DISCUSIÓN}

Los resultados obtenidos muestran que la escala ACDE evidencia 3 factores latentes que hacen referencia a las prácticas pedagógicas de Inclusión y Colaboración más un factor de actitud hacia la Democracia. Los tres factores son parsimoniosos, pues la estructura factorial se concentra en pocos factores, manteniendo una representación coherente de las dimensiones originales del instrumento y el marco teórico en general. La coherencia entre las dimensiones Pedagógica y Democrática del instrumento original con respecto a los tres factores latentes sugeridos por los índices de Horn y VSS, más el proceso de extracción y rotación factorial, permiten evidenciar que los factores de Inclusión y Colaboración componen en su conjunto la dimensión Pedagógica, mientras que el factor Democracia es equivalente a la dimensión Democrática, salvo el ítem "Utilizar sistemáticamente procesos deliberativos como parte del proceso educativo de los estudiantes" que, en la solución factorial, fue cargada al factor Inclusión, la que se mantuvo así, pues se consideró que los procesos deliberativos son una forma de incluir las distintas formas de ser y pensar en la actividad pedagógica (Castillo-Vargas y Mora-Castrillo, 2010). Respecto a la adecuación muestral, el índice $\mathrm{KMO}=0.827$ se puede considerar meritorio (Dziuban y Shirkey, 1974) y, en lo referente al ajuste del modelo, los índices son consistentes entre ellos y los resultados son similares a los evidenciados en escalas de autoeficacia aplicados a profesores chilenos (Covarrubias y Mendoza, 2016).

En particular, el factor Inclusión contempla las preguntas que hacen referencia a la capacidad autopercibida para promover y organizar un ambiente de aprendizaje inclusivo que tome en cuenta la realidad sociocultural de los estudiantes y promueva la reflexión sobre la exclusión de sus miembros. Este factor guarda relación con la propuesta de definición de convivencia de Fierro-Evans y Carbajal-Padilla (2019), los que, tras un estudio analítico y exploratorio actualizado del concepto de convivencia escolar, exponen como parte de la definición de convivencia las prácticas pedagógicas inclusivas y participativas. El factor Democracia reúne las preguntas referentes a la capacidad autopercibida de mantener una conducta democrática, flexible y comprensiva ante las dificultades generadas por situaciones de convivencia adversas. Este factor es congruente con la actitud de respeto y atención a las inquietudes e iniciativas de los estudiantes, es decir, en el sentido de Dewey (1995), hacer que el accionar docente refiera siempre a la acción de los estudiantes y que, a su vez, la acción de los estudiantes de pauta y dirección a la acción del profesor, incluso en situaciones adversas de convivencia. El factor Colaboración aborda principalmente la capacidad autopercibida de 
organizar los grupos de trabajo colaborativo en un contexto de heterogeneidad. Este factor guarda relación con el ideal democrático de Dewey (1995) en el sentido de que la colaboración es un producto de la democracia y no un fin en sí mismo, considerando que en la diversidad está la incitación a pensar y reflexionar democráticamente, por lo que la capacidad pedagógica de los futuros docentes para conformar grupos colaborativos con destrezas, habilidades y culturas diferentes es relevante para que sus estudiantes desarrollen conductas democráticas (Torres-Rivera y Florencio, 2019).

Sobre la relación entre las dimensiones Pedagógica (alfa de Cronbach $=0.9$ ) y Democrática (alfa de Cronbach $=0.844$ ) del instrumento original con respecto a los tres factores latentes, los resultados muestran que los factores de Inclusión y Colaboración componen en su conjunto la dimensión Pedagógica, mientras que el factor Democracia es equivalente a la dimensión Democrática, salvo el ítem "Utilizar sistemáticamente procesos deliberativos como parte del proceso educativo de los estudiantes" que, en la solución factorial, fue cargada al factor Inclusión, la que se mantuvo así, pues se consideró que los procesos deliberativos son una forma de incluir las distintas formas de ser y pensar en la actividad pedagógica (Castillo-Vargas y MoraCastrillo, 2010). Respecto a la adecuación muestral, el índice $\mathrm{KMO}=0.827$ se puede considerar meritorio (Dziuban y Shirkey, 1974) y, en lo referente al ajuste del modelo, los índices son consistentes entre ellos y los resultados son similares a los evidenciados en escalas de autoeficacia aplicados a profesores chilenos (Covarrubias y Mendoza, 2016).

Sobre las variables que caracterizan la muestra, se encontró una correlación negativa, aunque baja, entre la edad y los factores de autoeficacia del instrumento. La teoría de la autoeficacia docente indica que las experiencias pueden afectar negativa o positivamente la autoeficacia en el tiempo (Bandura, 1977). Al controlar la variable años de formación universitaria, la correlación entre la edad y los factores de autoeficacia manifiesta un aumento que evidencia la existencia de alguna causa temporal relacionada con la edad que disminuye la autoeficacia de los estudiantes y que no está directamente relacionada con la cantidad de años de formación. No se puede averiguar, mediante el diseño de esta investigación, la causa de esta disminución. Sin embargo, se puede hallar evidencia similar en el comportamiento de la autoeficacia para prácticas inclusivas (Dias, 2017), donde se encontró una correlación negativa de similar magnitud e igual signo entre ambas variables, aunque la muestra proviene de profesores en ejercicio.

En cuanto a la diferencia significativa de los puntajes de Educación Diferencial en el factor Inclusión con respecto a las otras disciplinas, los resultados también concuerdan con los de Dias (2017), donde se constató una mayor autoeficacia para las prácticas inclusivas en los profesores con formación inicial en educación especial respecto a los otros profesores. Cabe destacar que, la inclusión educativa abordada por el estudio de Días (2017) está más orientada a las necesidades educativas especiales, aunque contiene elementos que hacen referencia a lo disciplinar y tiene en consideración la heterogeneidad de los estudiantes.

Acerca de la diferencia entre los géneros femenino y masculino, en todos los factores se encontró un puntaje significativamente mayor para el género femenino. Según los estudios de Henson, Kogan y Vacha-Haase (2001), la autoeficacia basada en la teoría sociocognitiva constituye un constructo que mide la creencia de eficacia personal en desempeños específicos de un dominio, por lo que la desigualdad de la autoeficacia podría deberse a las diferencias en las fuentes de autoeficacia a la que están expuestos los estudiantes debido a su género, especialmente las experiencias vicarias dadas por los modelos de lo femenino y masculino del contexto cultural y educativo (Navarro et al., 2019). También, dicha distinción podría estar dada por la relación del género con las disciplinas que enmarcan cada profesión y que tienen influencia en los procesos de selección de las profesiones de los estudiantes (Asbún y Ferreira, 2004). Sin embargo, se tienen antecedentes de la relación de la autoeficacia docente con el clima escolar (Guangbao y Timothy, 2021; Mansor et al., 2021), por lo que podrían existir factores socioambientales relacionados a la convivencia que, por los límites de la presente investigación, hace necesario de que estos hechos deban ser confirmados en estudios posteriores.

Con respecto a la evaluación prospectiva mediante el instrumento propuesto, se puede evidenciar que la fiabilidad y la validez del instrumento muestra evidencia suficiente para explorar su utilización en otras muestras de composición similar de profesores en formación. En relación con la validación de instrumentos, Charlton et al. (2021), tras una revisión de 26 intervenciones en el clima escolar con perspectiva global (School-wide climate), concluye que es necesario proveer mayor sistematización y validación de los instrumentos psicométricos, pues existe una inconsistencia conceptual en el área que dificulta su evaluación y la comparación de los hallazgos. Esto también es relevante en la FID, pues los instrumentos psicométricos de autoeficacia son más efectivos, mientras más específico es el dominio que evalúan. Además, la autoeficacia es un constructo con capacidad predictiva del desempeño que no se basa directamente en los conocimientos y habilidades de las personas (Dunn y Rakes, 2011), por lo que esta característica permite evaluar la preparación de los profesores para enfrentar los desafíos de la convivencia escolar en cualquier etapa de su formación, con tal de realizar las intervenciones necesarias que apunten a aumentar las 
probabilidades de éxito en el desempeño futuro. Adicionalmente, la autoeficacia de los profesores es influida por factores personales y socioambientales, tales como la propia concepción del rol docente en la convivencia escolar, la disciplina de especialización y el clima escolar (Charlton et al. 2021, Erlich y Gindi, 2019), por lo que poseer un instrumento de autoeficacia en dominios específicos, permite evaluar la relación de estos factores y su efecto en la autoeficacia en los dominios abordados por la escala ACDE (inclusión, democracia y colaboración).

Para finalizar, es importante indicar que la investigación tiene limitaciones con respecto al tamaño de la muestra utilizada. Aunque se puede considerar que la muestra es suficiente para un enfoque exploratorio, se recomienda 200 participantes o más para confirmar los constructos del instrumento mediante un análisis confirmatorio. Sin embargo, los índices de fiabilidad y adecuación del modelo indican que los factores encontrados explican con suficiencia el constructo analizado.

\section{CONCLUSIONES}

De acuerdo con el instrumento propuesto, los resultados evidenciados y la discusión, se pueden extraer las siguientes conclusiones: 1) el instrumento Autoeficacia Docente para la Convivencia Escolar (ADCE) posee tres variables latentes organizadas en los factores de Colaboración, Inclusión y Democracia; 2) la solución factorial muestra un ajuste adecuado, una alta fiabilidad y es parsimonioso con el constructo teórico; 3) los factores para la autoeficacia docente en convivencia escolar presentan diferencias significativas según el género y la formación para la educación especial; 4) existe una correlación significativa e inversamente proporcional de los factores de autoeficacia con respecto a la edad de los participantes; 5) es posible utilizar la escala ADCE para evaluar, de forma prospectiva, la autoeficacia para la convivencia escolar de profesores en formación en los dominios acotados de la inclusión, colaboración y democracia.

\section{REFERENCIAS}

Asbún, C., y Ferreira, Y., Autoeficacia Profesional y Género en Adolescentes de Cuarto de Secundaria de la Zona Sur de la Ciudad de la Paz, Ajayu Órgano de Difusión Científica del Departamento de Psicología UCBSP, 2(1), 12-20 (2004).

Ascorra, P., López, V., y otros 4 autores, Significados Atribuidos a la Convivencia Escolar por Equipos Directivos, Docentes y Otros Profesionales de Escuelas Chilenas, https://doi.org/https://doi.org/10.7764/psykhe.27.1.1214, Psykhe (Santiago), 27(1), 1-12 (2018).

Bandura, A., Self-efficacy: Toward a Unifying Theory of Behavioral Change, https://doi.org/doi:10.1037/0033295x.84.2.191, Psychological Review, 84(2), 191-215 (1977).

Castillo-Vargas, A., y Mora-Castrillo, C., Estrategia Pedagógica para la Deliberación y el Razonamiento Sociomoral en Jóvenes de Secundaria, http://dx.doi.org/10.15517/ap.v23i110.13, Actualidades en Psicología, 23(110), 103-129 (2010).

Chaparro, A. A., Caso, J., Fierro, M. C., y Díaz, C., Desarrollo de un Instrumento de Evaluación Basado en Indicadores de Convivencia Escolar Democrática, Inclusiva y Pacífica, Perfiles Educativos, 37(149), $20-41$ (2015).

Covarrubias, C. G., y Mendoza, M. C., Adaptación y Validación del Cuestionario Sentimiento de Autoeficacia en una Muestra de Profesores Chilenos, https://doi.org/http://dx.doi.org/10.11144/Javeriana.upsy15-2.avcs, Universitas Psychologica, 15(2), 97-108 (2016).

Covarrubias, C. G., y Mendoza, M. C., La Teoría de Autoeficacia y el Desempeño Docente: El Caso de Chile, Estudios Hemisféricos y Polares, 4(2), 107-123 (2013).

Charlton, C. T., Moulton, S., Sabey, C. V., y West, R., A., Systematic Review of the Effects of Schoolwide Intervention Programs on Student and Teacher Perceptions of School Climate, https://doi.org/10.1177/1098300720940168, Journal of Positive Behavior Interventions, 23(3), 185-200 (2021).

Delors, J., La Educación Encierra un Tesoro, UNESCO (1996).

Dewey, J., Democracia y Educación: Una Introducción a la Filosofía de la Educación, 3ra. Edición, Ediciones Morata, Madrid, España (1998).

Dias, P. C., A Autoeficácia dos Professores para a Implementação de Práticas Inclusivas: Contributos para uma Reflexão sobre a Inclusão Educative, https://doi.org/10.1590/s0104-40362017000100001, Ensaio: Avaliação e Políticas Públicas em Educação, 25(94), 7-25 (2017).

Dunn, K. E., y Rakes, G. C., Teaching Teachers: An Investigation of Beliefs in Teacher Education Students, https://doi.org/10.1007/s10984-011-9083-1, Learning Environments Research, 14, 39-58 (2011).

Dziuban, C. D., y Shirkey, E. C., When is a Correlation Matrix Appropriate for Factor Analysis? Some Decision Rules, https://doi.org/10.1037/h0036316, Psychological Bulletin, 81(6), 358 (1974). 
Erlich, R. R., y Gindi, S., Are Civics Teachers Different from Teachers of Other Disciplines in their Handling of Controversial Issues?, https://doi.org/10.1177/1746197918767082, Education, Citizenship and Social Justice, 14(2), 118-130 (2019).

Fierro-Evans, C., y Carbajal-Padilla, P., Convivencia Escolar: Una Revisión del Concepto, https://doi.org/10.5027/psicoperspectivas-vol18-issue1-fulltext-1486, Psicoperspectivas Individuo y Sociedad, 18(1), 1-14 (2019).

Gibson, S., y Dembo, M. H., Teacher Efficacy: A Construct Validation, https://doi.org/10.1037/0022-0663.76.4.569, Journal of Educational Psychology, 76(4), 569-582 (1984).

Guangbao, F., y Timothy, T., Investigating the Associations of Constructivist Beliefs and Classroom Climate on Teachers' Self-Efficacy Among Australian Secondary Mathematics Teachers, https://doi.org/10.3389/fpsyg.2021.626271, Frontiers in Psychology, 12 (2021).

Henson, R. K., Kogan, L. R., y Vacha-Haase, T., A Reliability Generalization Study of the Teacher Efficacy Scale and Related Instruments, https://doi.org/10.1177/00131640121971284, Educational and Psychological Measurement, 61(3), 404-420 (2001).

Lazarides, R., Fauth, B., Gaspard, H., y Göllner, R., Teacher Self-efficacy and Enthusiasm: Relations to Changes in Student-perceived Teaching Quality at the Beginning of Secondary Education, https://doi.org/https://doi.org/10.1016/j.learninstruc.2020.101435, Learning and Instruction, 73, (2021).

Loubiès, L., Valdivieso, P., y Vásquez, C., Challenges to Initial Teacher Training in School Coexistence, https://dx.doi.org/10.4067/S0718-07052020000100223, Estudios Pedagógicos, 46(1), 223-239 (2020).

Milson, A. J., y Mehlig, L. M., Elementary School Teachers' Sense of Efficacy for Character Education, https://doi.org/10.1080/00220670209598790, The Journal of Educational Research, 96(1), 47-53 (2002).

Muñoz, P. E., Casas, J. A., y otros 4 autores, Validation and Cross-Cultural Robustness of the School-wide Climate Scale (SCS) across Spanish and Chilean Students, https://doi.org/doi:10.1016/j.stueduc.2018.01.002, Studies in Educational Evaluation, 56, 182-188 (2018).

Navarro, G., Flores-Oyarzo, G., y González, M. G., Diferencias por Sexo en el Nivel de Autoeficacia Percibida en una Muestra de Estudiantes de la Provincia de Concepción ¿Qué Papel Juegan los Roles de Género en la Educación?, Revista de Educación Inclusiva, 12(1), 205-224 (2019).

Pajares, F., y Schunk, D. H., Self and Self-belief in Psychology and Education: A Historical Perspective, Improving Academic Achievement Impact of Psychological Factors on Education, https://doi.org/10.1016/B978-0120644551/50004-X, Academic Press, Amsterdam, 3-20 (2002).

Pedraja-Rejas, L., Araneda-Guirriman, C., Rodríguez-Ponce, E., y Rodríguez-Ponce, J., Calidad en la Formación Inicial Docente: Evidencia Empírica en las Universidades Chilenas, https://dx.doi.org/10.4067/S0718-50062012000400003, Formación Universitaria, 5(4), 15-26 (2021).

Retuert, G., y Castro, P. J., Teorías subjetivas de profesores acerca de su rol en la construcción de la convivencia escolar, https://dx.doi.org/10.4067/S0718-65682017000100321, Polis (Santiago), 16(46), $321-345$ (2017).

Revelle, W., y Rocklin, T., Very Simple Structure: An Alternative Procedure for Estimating the Optimal Number of Interpretable Factors, https://doi.org/10.1207/s15327906mbr1404_2, Multivariate Behavioral Research, 14(4), 403-414 (1979)

Torres-Rivera, A., y Florencio, R., Aprender a Convivir en Educación Superior desde la Práctica Docente, para una Sociedad Democrática, https://dx.doi.org/10.4067/S0718-5006201900020005, Formación Universitaria, 12(2), 51-62 (2019).

Tschannen-Moran, M., y Hoy, A. W., Teacher Efficacy: Capturing an Elusive Construct, https://doi.org/10.1016/S0742051X(01)00036-1, Teaching and Teacher Education, 17(7), 783-805 (2001).

Velicer, W. F., The Relation Between Factor Score Estimates, Image Scores, and Principal Component Scores, https://doi.org/10.1177/001316447603600114, Educational and Psychological Measurement, 36(1), 149-159 (1976). 
